\title{
DIFERENCIAS DE GÉNERO EN EL ACOSO PSICOLÓGICO EN EL TRABAJO: UN ESTUDIO EN POBLACIÓN ESPAÑOLA
}

\author{
Bernardo Moreno Jiménez \\ Alfredo Rodríguez Muñoz \\ Eva Garrosa Hernández \\ Maria Eugenia Morante Benadero \\ Raquel Rodríguez Carvajal ${ }^{\mathscr{E}}$
}

\begin{abstract}
RESUMO. En los últimos años, el acoso psicológico en el trabajo (bullying o mobbing) ha recibido una atención constante y ha sido reconocido como un importante problema social. El objetivo de este estudio consiste en analizar de forma exploratoria si existen diferencias de género tanto en el acoso psicológico como en sus dimensiones. Los datos fueron recogidos con el cuestionario de "Acoso Psicológico en el Trabajo" (Moreno-Jiménez, Rodríguez-Muñoz, Garrosa, Morante \& Rodríguez, 2004), en una muestra de 103 trabajadores de la Comunidad de Madrid. Los resultados mostraron diferencias significativas entre hombres y mujeres en Acoso Psicológico, Aislamiento Social y Desacreditación. Estos datos apoyan la hipótesis de que el hecho de ser mujer supone un riesgo adicional para sufrir acoso psicológico.
\end{abstract}

Palabras clave: acoso psicológico en el trabajo, diferencias de género, desequilibrio de poder.

\section{GENDER DIFFERENCES IN WORKPLACE BULLYING: A STUDY IN A SPANISH SAMPLE}

\begin{abstract}
Recently, workplace bullying has received increased attention and has been recognised as an important social problem. The aim of the present study was to analyze in an exploratory way whether exist gender differences as much on bullying as on its dimensions. Data were collected with the "Bullying at Work Questionnaire" (Moreno-Jiménez, RodríguezMuñoz, Garrosa, Morante \& Rodríguez, 2004), in a sample of 103 employees of the Community of Madrid. The results showed significant differences between men and women in Bullying, Social Isolation and Discredit. These data support the hypothesis that the fact of being women supposes an additional risk of being bullied at work.
\end{abstract}

Key words: bullying, gender differences, imbalance of power.

En los últimos años, el acoso psicológico (mobbing o bullying) ha recibido una creciente atención por parte de la comunidad científica como un importante problema social en las organizaciones y se ha convertido en un tópico de investigación de amplio interés en numerosos países occidentales, preferentemente europeos y norteamericanos
(Björkqvist, Österman \& Hjelt-Bäck, 1994a; Einarsen, Raknes \& Matthiesen, 1994; Rayner, Hoel \& Cooper, 2002; Leymann, 1986; Niedl, 1995; Salin, 2001; Vartia, 1996; Zapf, Knorz \& Kulla, 1996). Este incremento de la atención parece debido a la constatación de la amplitud y profundidad de sus consecuencias. Se ha demostrado que el acoso

\footnotetext{
* Doctor en Psicología. Profesor Titular del Departamento de Psicologia Biológica y de la Salud de la Universidad Autónoma de Madrid.

\# Psicólogo. Master en Prevención de Riesgos Laborales, Universidad Autónoma de Madrid.

II Doctora en Psicología, Universidad Autónoma de Madrid.

x Psicóloga. Master en Prevención de Riesgos Laborales, Universidad Autónoma de Madrid.

Æ Psicóloga. Master en Psicología Clínica y de la Salud, Universidad Autónoma de Madrid.
} 
psicológico puede causar severas consecuencias sobre la salud de los trabajadores, e inciden en ellos de forma muy variada; a nivel individual, las víctimas presentan un amplio número de síntomas de estrés (Brodsky, 1976; Einarsen, Raknes \& Matthiesen, 1994; Mikkelsen \& Einarsen, 2001), y elevados síntomas psicosomáticos así como enfermedades físicas y baja satisfacción laboral (Groeblinghoff \& Becker, 1996; Niedl, 1995; Zapf, Knorz \& Kulla, 1996). En el ámbito organizacional, se ha comprobado que el acoso laboral está asociado con altas tasas de absentismo, numerosos intentos de abandonar la organización y con la rotación laboral (Leymann, 1996; Rayner, 1997). Las amplias consecuencias resultantes del acoso psicológico han convertido este fenómeno en un tema de gran interés público y es considerado como una de las formas más severas de estrés psicosocial en el trabajo (Niedl, 1995).

El acoso psicológico en el trabajo es concebido como un proceso que, en primer lugar, alude a la violencia psíquica, directa e indirecta, que se puede ejercer sobre alguien; en segundo lugar, se refiere a un tipo de conducta que se ejerce de forma continuada en el tiempo; y, en tercer lugar, describe conductas de marginación, discriminación y vejación que pueden ser abiertas, dirigidas a la totalidad de la persona y a su hundimiento humano como persona (MorenoJiménez, Garrosa, Galindo, San Julián, Rodríguez, Morante, \& Losada, 2002). A pesar de que el acoso psicológico es un fenómeno antiguo, no ha sido descrito ni sistemáticamente investigado hasta comienzos de los años ochenta. Aunque anteriormente ya había sido abordado (Brodsky, 1976), no fue tratado como objeto especifico de estudio científico en el ámbito laboral hasta 1984, cuando se publicó el informe del National Board of Occupational Safety and Health de Estocolmo (Leymann \& Gustafsson, 1984). Leymann (1996) planteó que “el mobbing consiste en la comunicación hostil y sin ética, dirigida de manera sistemática por uno o varios individuos contra otro, que es así arrastrado a una posición de indefensión y desvalimiento, y activamente mantenido en ella (...) a causa de la elevada frecuencia y duración de la conducta hostil, este maltrato acaba por producir una considerable miseria mental, psicosomática y social". De acuerdo con esta definición, para concebir una acción como acoso psicológico esta debe cumplir al menos dos criterios temporales: deben tener lugar "de manera frecuente (por lo menos una vez a la semana) y durante largo tiempo (por lo menos seis meses)". Además, el poder, o más concretamente las diferencias de poder, son vistas como un elemento central en la mayoría de las definiciones de acoso. En otras palabras, el acoso es definido como un conflicto en el que la victima no tiene capacidad para defenderse (Hoel \& Cooper, 2000; Vartia, 1996).

\section{APROXIMACIONES TEÓRICAS AL ACOSO PSICOLÓGICO}

Las aproximaciones teóricas al acoso psicológico han sido un tema candente y controvertido tanto en los medios de comunicación como entre la comunidad científica. Se han llevado a cabo numeroso intentos para explicar la aparición del acoso que según Einarsen (2000) se dividen en tres bloques: 1) explicación en base a características del entorno laboral y social., 2) en base a las características inherentes a las relaciones interpersonales y 3) en función de la personalidad, tanto de las víctimas como de los acosadores.

El modelo que mayor atención académica ha recibido y sobre el que existe mayor consenso es el que enfatiza la importancia de los factores organizacionales y psicosociales como variables causales, tales como un mal ambiente en el trabajo o un pobre contenido de la tarea. De acuerdo con este tipo de aproximación se puede afirmar que el acoso psicológico se encuentra fuertemente relacionado con numerosos elementos del ambiente y la organización del trabajo, como por ejemplo el estilo de liderazgo (Einarsen, Raknes \& Matthiesen, 1994; Hoel \& Cooper, 2000), el tipo de organización (Thylefors, 1987), las demandas laborales, el control sobre la tarea (Zapf, Knorz \& Kulla, 1996) así como el clima social y la comunicación interna (Einarsen, Raknes \& Matthiesen, 1994; Moreno-Jiménez, RodríguezMuñoz, Morante \& Rodríguez, 2004). En esta misma línea psicosocial, ciertas políticas y prácticas empresariales tales como los despidos, reestructuración, remodelación, reducción de plantilla, fusiones etc., se han relacionado con la aparición del acoso laboral (Baron \& Neuman, 1998; McCarthy, Sheehan \& Wilkie, 1995).

El enfoque de aproximación psicosocial ha sido fuertemente defendido por Leymann (1992) quien considera que los factores de personalidad son irrelevantes para el estudio del acoso psicológico, y que las condiciones de trabajo son la causa única del acoso. Sin embargo, los resultados de otras investigaciones sugieren que es bastante improbable que el acoso pueda ser explicado exclusivamente en términos de condiciones del trabajo.

Por otra parte, las aproximaciones centradas en las características inherentes a las relaciones humanas en la organización enfatizan el conflicto como normal 
en las interacciones cotidianas en las organizaciones. Algunos autores (Brodsky, 1976) defienden que la competición es consustancial a todos los grupos sociales, étnicos y raciales en la medida que se produce un constante proceso de evaluación de ajuste entre uno mismo y los demás con el fin de establecer el lugar que cada uno ocupa en la jerarquía.

Numerosos investigadores han tratado de identificar las variables de personalidad asociadas con el acoso psicológico. Dentro de esta perspectiva, se ha señalado que los resultados de los estudios apuntan que algunas víctimas del acoso laboral son más sensibles al mismo o reaccionan de un modo más dramático que otras ante las situaciones de acoso (Matthiesen \& Einarsen, 2001). Del mismo modo, algunos autores describen a las víctimas como paranoicas, rígidas, compulsivas (Brodsky, 1976), con falta de competencias sociales (Zapf \& Einarsen, 2003) y con mayores puntuaciones en neuroticismo (Vartia, 1996). La hipótesis de la personalidad propone que algunas personas tienen mayores riesgos de convertirse en víctimas de acoso, como por ejemplo los empleados sumisos o los trabajadores provocativos (Aquino \& Bradfield, 2000). En la misma línea, según los planteamientos de Zapf y Einarsen (2003), parece que determinadas personas parecen tener mayor riesgo de padecer acoso. En este sentido, se ha señalado que la aparición del acoso psicológico esta relacionada con el género y que el hecho de ser mujer es un riesgo potencial para sufrir acoso. Sin embargo, existen discrepancias en la literatura al respecto. De los diferentes estudios, algunos mostraron que los hombres y las mujeres son acosados en igual medida (Einarsen \& Skogstad, 1996; Hoel \& Cooper, 2000; Vartia, 1996), mientras que otros señalan que las mujeres son acosadas con mayor frecuencia (Björkqvist, Österman \& Lagerspetz, 1994b; Salin, 2003a). En el estudio de Björkqvist, Österman y Lagerspetz (1994b), el 25\% de los participantes mencionaron el género como una posible razón para el acoso. Por contra, Hoel y Cooper (2000) encontraron que los hombres eran expuestos más frecuentemente a conductas negativas en el trabajo que las mujeres.

Recientemente, algunos autores como Einarsen (2000), han planteado la existencia de diferencias organizativas transculturales como factor explicativo de estas divergencias. En su revisión sobre el estado de la cuestión del acoso, señaló que la cultura organizativa escandinava es predominantemente igualitaria, y el hecho de que la mayoría de estudios sobre mobbing se hayan llevado a cabo en dichos países, podría explicar que se haya encontrado una victimización similar entre los hombres y las mujeres.

El objetivo del presente trabajo consiste en analizar, de forma exploratoria, las diferencias de género en el acoso psicológico y las dimensiones que lo integran.

\section{MÉTODO}

\section{Sujetos}

La muestra estuvo formada por trabajadores del Sector de Transportes y Comunicaciones de la Comunidad de Madrid. El número de trabajadores que respondieron al estudio fue de 103 sobre un total de 330 cuestionarios distribuidos, lo que representa una tasa de respuesta del $31,21 \%$. Esta baja tasa de respuesta ha sido constatada como normal en investigaciones sobre el acoso psicológico en vista a la sensibilidad del tema que se pregunta (Björkqvist, Österman \& Lagerspetz, 1994b). La muestra del presente estudio incluye un 50,5\% de mujeres, con una edad media de 34 años y una desviación típica de 8,00 . El $44,1 \%$ de los trabajadores son operarios y el $41,2 \%$ trabajan en horario de turno partido. La participación fue voluntaria y toda la información recibida fue totalmente confidencial.

\section{Instrumentos}

En este trabajo se utilizó el "Cuestionario de Acoso psicológico en el Trabajo" (Moreno-Jiménez, Rodríguez-Muñoz, Garrosa, Morante \& Rodríguez, 2004). El cuestionario contiene dos secciones netamente diferenciadas, la primera incluye variables sociodemográficas y laborales y la segunda cuestiones relativas a las formas de acoso y los factores organizacionales.

La primera parte incluye variables básicas como edad y género que parecen estar relacionadas con conductas de acoso (Piñuel, 2001), a ellas se añadieron otras variables laborales que en las entrevistas habían aparecido como probablemente relacionadas con las tasas de acoso (años de experiencia laboral, experiencia en el sector, experiencia en el mismo puesto, situación laboral, sector en el que trabaja, cargo que ocupa en la empresa, tipo de contrato, turno, tipo empresa, tipo convenio, horario y turno). La categorización de las respuestas varía en función de la variable, y en el caso del tipo de contrato, que es una de las variables analizadas específicamente en el presente trabajo, la categorización de las respuestas se distribuye de la siguiente manera: 1 ("Eventual"), 2 ("Por obra o Empresas de Trabajo Temporal"), 3 ("Indefinido") y 4 ("Funcionario"). Por su parte, el nivel de estudios se divide en 1 ("Elementales"), 2 ("Medios") y 3 ("Superiores"). 
La segunda parte consta de dos escalas:

- Escala de acoso psicológico compuesta por las dimensiones de aislamiento social, desacreditación, exigencia extrema y consecuencias. Esta escala consta de 15 ítems que recogen conductas típicas de situaciones de acoso, respecto a las cuales el sujeto debe indicar el grado en que ha padecido las conductas en una escala de 0 ("Nunca") a 4 ("Continuamente").

- Escala de factores organizacionales compuesta por dos factores; Política organizacional e Información y actuaciones ante el acoso. El factor Política organizacional hace referencia a estilos de liderazgo, toma de decisiones y comunicación. El factor Información y actuaciones alude referencia a la información y acciones que se llevan a cabo en la organización en el tema de acoso psicológico. El formato de respuesta se recoge en una escala de 0 ("Nunca") a 4 ("Continuamente").

El cuestionario cuenta con un índice global de consistencia de 0,94. Además, todas las escalas que componen el cuestionario superan el criterio de 0,70 recomendados (Nunnaly \& Berstein, 1994). En la validación factorial, todos los ítems presentaron cargas factoriales superiores a 0,55 , criterio a partir del cual son consideradas como buenas (Comrey, 1973).

\section{Procedimiento}

En el contexto del Plan Director en Prevención de Riesgos Laborales de la Comunidad Autónoma de Madrid, el Instituto Regional de Seguridad y Salud en el Trabajo y la Unión General de Trabajadores (UGT) de Madrid acordaron como uno de los objetivos el estudio del acoso psicológico en el trabajo. Para ello se decidió realizar una revisión teórica y un estudio empírico exploratorio sobre el alcance del acoso psicológico en el Sector de Transportes y Comunicaciones, del que había datos que sugerían un alto porcentaje de trabajadores acosados. Para ello se procedió a revisar diferentes instrumentos de evaluación y a mantener diez entrevistas en profundidad con trabajadores del sector para detectar indicadores del acoso psicológico.

Antes de la aplicación de la prueba se realizó un estudio piloto con 30 sujetos, con el objetivo de analizar posibles dificultades en la aplicación de la misma. La redacción de algunos ítems fue modificada para hacerlos más fácilmente comprensibles. Una vez elaborado el instrumento se aplicó a los trabajadores a través de los delegados de prevención de la propia empresa, compañeros laborales de los trabajadores directamente encargados de aspectos relacionados con la Prevención de Riesgos Laborales. La entrega y recogida de los cuestionarios fue realizada a través de los delegados de prevención, recordándoles que debía responderse de forma individual y que era anónimo.

\section{RESULTADOS}

Aproximadamente el $26 \%$ de los sujetos informaron haber padecido una o más conductas negativas al menos una vez a la semana, durante los últimos seis meses. De estas víctimas, el 52,5\% fueron acosados exclusivamente por sus superiores, el $18,4 \%$ por sus compañeros de trabajo, mientras que el $7,1 \%$ de las víctimas informaron haber padecido acoso tanto por sus superiores como por sus compañeros. Para analizar si existían diferencias de género en el acoso psicológico y sus formas, se procedió a realizar una prueba t de student para muestras independientes. Para ello se tomó como variables independientes la variable género (1. Hombre, 2. Mujer). Como variables dependientes se tomaron tanto el acoso psicológico global como cada de las dimensiones que lo componen (Aislamiento social, Exigencia extrema, Desacreditación y Consecuencias del acoso). Los descriptivos de ambos grupos se presentan en la Tabla 1:

Tabla 1. Estadísticos Descriptivos

\begin{tabular}{lccc}
\hline Variables & Género & Media & D.T. \\
\hline Aislamiento social & Masculino & 1,3612 & 0,8937 \\
& Femenino & 1,9830 & 1,1535 \\
Exigencia extrema & Masculino & 1,5629 & 0,8871 \\
& Femenino & 1,7700 & 1,1360 \\
Desacreditación & Masculino & 1,1359 & 0,7221 \\
& Femenino & 1,5935 & 1,0132 \\
Consecuencias & Masculino & 1,2500 & 0,9589 \\
& Femenino & 1,4375 & 1,1788 \\
Acoso Psicológico Global & Masculino & 1,3535 & 0,8060 \\
& Femenino & 1,7941 & 1,0573 \\
\hline
\end{tabular}

El contraste de medias entre ambos grupos esta expuesto en la Tabla 2:

Tabla 2. Prueba T de Student

\begin{tabular}{lccccc}
\hline & \multicolumn{5}{c}{ Prueba T para la igualdad de medias } \\
\cline { 2 - 6 } & $\mathbf{t}$ & gl & Sig, & $\begin{array}{c}\text { Diferencia Error típ, de } \\
\text { de medias }\end{array}$ & la diferencia \\
\hline Aislamiento social & $-3,002$ & 92,127 & 0,003 &,- 6218 &, 2071 \\
Exigencia extrema & $-1,012$ & 92,422 & 0,314 &,- 2071 &, 2046 \\
Desacreditación & $-2,547$ & 86,868 & 0,013 &,- 4577 &, 1797 \\
Consecuencias & $-0,848$ & 89,663 & 0,399 &,- 1875 &, 2212 \\
Acoso Psicológico & $-2,293$ & 89,312 & 0,024 &,- 4406 &, 1922 \\
Global & & & & & \\
\hline
\end{tabular}

En el análisis descriptivo, se observó que en el acoso psicológico global $\left(M_{\text {Hombre }}=1,353\right.$; $\left.M_{\text {Mujer }}=1,794\right)$ las mujeres obtuvieron una media 
significativamente superior a la de los hombres. Mientras que en el análisis por dimensiones se aprecia un patrón similar, obteniendo las mujeres mayores puntuaciones en todas las dimensiones que componen el acoso. La prueba t muestra diferencias significativas en las dimensiones Aislamiento social $(p<0,01)$ y Desacreditación $(p<0,05)$. Del mismo modo, las diferencias en las puntuaciones entre hombre $\mathrm{y}$ mujeres en acoso psicológico resultaron significativas $(p<0,05)$. Los datos se muestran en forma gráfica en la figura 1 .

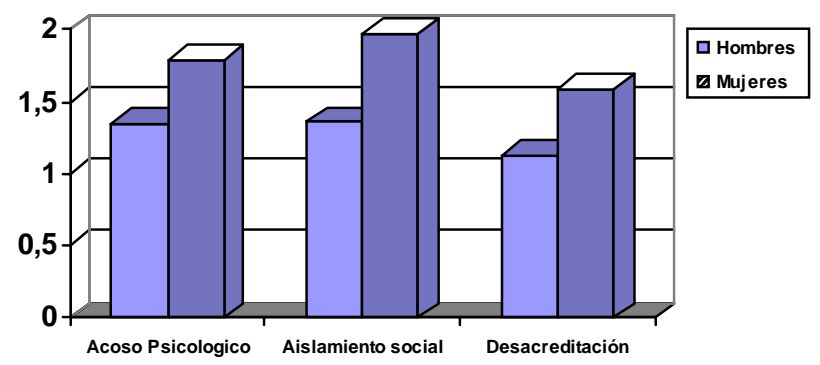

Figura 1 - Diferencias significativas en Acoso psicológico y sus dimensiones

En un análisis más pormenorizado, se observó que la conducta de acoso que más habían recibido las mujeres era el haber sido controlado y/o vigilado en extremo, ítem en el cual $\left(M_{\text {Hombre }}=1,48\right.$; $\left.M_{\text {Mujer }}=2,28\right)$ existían claras diferencias significativas con los hombres $(p<0,01)$. En el mismo sentido, las mujeres habían sido ignoradas con mayor frecuencia $\left(M_{\text {Hombre }}=1,34 ; \quad M_{\text {Mujer }}=1,90\right)$, habían recibido más respuestas hostiles a sus comentarios y preguntas $\left(M_{\text {Hombre }}=1,38 ; M_{\text {Mujer }}=2,10\right)$ y sus preguntas habían sido ignoradas $\left(M_{\text {Hombre }}=1,55 ; M_{\text {Mujer }}=2,14\right)$; en todas estas conductas las diferencias con los hombres resultaron significativas. En los análisis previos no se encontraron diferencias significativas en cuanto a la dimensión exigencia extrema, pero en el ítem "Me recuerdan constantemente cualquier error que cometo, por pequeño que sea", existen diferencias significativas $(p<0,05)$, obteniendo las mujeres una mayor puntuación $\left(M_{\text {Hombre }}=1,39 ; M_{\text {Mujer }}=2,00\right)$. El análisis de los ítems de la dimensión desacreditación muestra que las mujeres padecen mayores criticas continuadas e injustificadas sobre su trabajo $\left(M_{\text {Hombre }}=1,33 ; M_{\text {Mujer }}=1,82\right)$, son más frecuentemente víctimas de comentarios malintencionados de compañeros o superiores $\left(M_{\text {Hombre }}=1,29 ; M_{\text {Mujer }}=1,92\right)$ $\mathrm{y}$ son objeto de insultos u ofensas sobre su persona, actitudes o su vida privada en mayor proporción que los hombres $\left(M_{\text {Hombre }}=0,98 ; M_{\text {Mujer }}=1,51\right)$, resultando estas diferencias significativas.
Complementariamente, en el análisis del proceso de acoso psicológico en función del género, cabe preguntarse si los procesos predictivos son similares para hombres y mujeres. Para ello, se llevó a cabo un análisis de regresión por pasos separado para cada sexo o género. Se tomaron como variables independientes las variables organizacionales (política organizacional e información y actuaciones ante el acoso), el tipo de contrato laboral, el nivel educativo y la experiencia laboral. Como variable dependiente se tomó el acoso psicológico global. Los datos se encuentran en la Tabla 3.

Tabla 3. Análisis de Regresión del Acoso Psicológico

\begin{tabular}{llcccc}
\hline Grupo & Variables Predictoras & Paso & $\mathbf{B}$ & $\mathbf{R}^{\mathbf{2}}$ & $\mathbf{F}$ \\
\hline Hombres & Información y Actuaciones & 1. & $-0,515^{* *}$ &, 266 & $11,569^{* *}$ \\
& Política Organizacional & 2. & $0,675^{* *}$ &, 573 & $20,778^{* *}$ \\
& Nivel de Estudios & 3. & $-0,289^{*}$ &, 652 & $18,733^{* *}$ \\
& Tipo de Contrato & 4. & $-0,254^{*}$ &, 713 & $18,004^{* *}$ \\
& & & & & \\
Mujeres & Nivel de Estudios & 1. & $-0,588^{* *}$ &, 346 & $17,484^{* *}$ \\
& Experiencia Laboral & 2. & $-0,370^{*}$ &, 469 & $14,134^{* *}$ \\
& Política Organizacional & 3. & $0,329^{*}$ &, 565 & $13,435^{* *}$ \\
& Tipo de contrato & 4. & $-0,248^{*}$ &, 623 & $12,387^{* *}$ \\
\hline
\end{tabular}

$* p<.05 ; * * p<.01$.

Como se observa, existen claras diferencias entre hombres y mujeres en los factores predictivos del acoso. En ambos grupos las variables con mayor capacidad predictiva son diferentes, mientras que en el grupo de los hombres la variable con mayor capacidad predictiva es la información y actuaciones ante el acoso, en el grupo de las mujeres el nivel de estudios es el factor que mayor varianza explica en la aparición del acoso. En el primer grupo, son los factores organizacionales (política organizacional e información y actuaciones) los que mayor varianza explican, ambos conjuntamente dan cuenta del 57,3\% de la varianza total del acoso en el grupo de los hombres. Por contra, en el grupo de las mujeres, parecen ser las competencias personales las variables que mayor capacidad predictiva tienen, ya que el nivel de estudios, junto con la experiencia laboral, explican el $46,9 \%$ de la varianza total del acoso en el grupo de mujeres.

\section{DISCUSIÓN Y CONCLUSIONES}

Los resultados obtenidos parecen indicar, de acuerdo con la hipótesis supuesta inicialmente, que las mujeres están expuestas a mayores conductas de acoso psicológico, puntuando mas alto que los hombres en acoso, así como en todas las dimensiones que componen el síndrome. Los resultados que aquí se 
presentan se hallan en consonancia con anteriores trabajos que indican que la prevalencia del acoso es mayor en mujeres que en hombres y que el hecho de ser mujer parece ser un riesgo potencial a la hora de padecer acoso. En un estudio llevado a cabo por Björkqvist, Österman y Lagerspetz (1994b) entre trabajadores finlandeses, encontraron diferencias significativas en la prevalencia del acoso entre mujeres y hombres, siendo las primeras más acosadas significativamente. Igualmente, los datos indican que existen diferencias en los factores etiológicos del síndrome entre hombres y mujeres. En el grupo masculino son los factores organizacionales los que parecen estar influyendo en mayor medida en la aparición del acoso, mientras que en el grupo de mujeres casi la mitad de la varianza es explicada por las competencias personales. Parece que las mujeres con un menor nivel de estudios, y con menor experiencia laboral tienen mayor riesgo de sufrir acoso laboral.

El género es un elemento que ha sido asociado frecuentemente con deficiencias de poder (Halford \& Leonard, 2001; Scott, 1988). Conceptualmente, las diferencias de poder entre la víctima y el acosador son esenciales para la experiencia de victimización (Niedl, 1995). De hecho ya en sus primeras publicaciones Leymann (1986) describió el acoso psicológico como una situación donde las víctimas tienen dificultades para defenderse y en las cuales las diferencias de poder jugaban un rol muy relevante. En años posteriores Leymann (1996) apuntaba que el acoso puede ser consecuencia de un mercado laboral segregado en razón del género. Igualmente, Hoel y Salin (2003) enfatizan las diferencias de poder como un factor crucial en la aparición del acoso psicológico.

Como afirma Einarsen (2000), las diferencias de poder o también conocidas como "distancia de poder" (Hofstede, 1980) y que se definen como la diferencia de influencia entre dos personas, varía a través de las culturas. Esto tiene importantes implicaciones pues como comentábamos en la introducción, tradicionalmente, la mayoría de los estudios se han llevado a cabo en los países escandinavos. En este sentido, Noruega, Suecia y Finlandia, han sido identificadas como culturas con relativamente pequeñas diferencias de poder y status entre los individuos en diferentes posiciones formales $\mathrm{e}$ informales dentro de las organizaciones. Estos países pueden de esta forma tener unas diferencias de género menores comparados con países como Francia o España que han sido identificados como países con mayores desequilibrios de poder en las organizaciones (Hofstede, 1980). Este mismo autor, afirma que los países escandinavos poseen culturas más igualitarias y más orientadas hacia los valores femeninos. Estas culturas prescriben a sus miembros a no ser agresivos, dominantes y asertivos en sus relaciones interpersonales. A la hora de interpretar las diferencias de género no se debe olvidar que, en muchos casos, el entorno de trabajo facilita la aparición de este tipo de discriminación debido a las desigualdades existentes en el mismo. En este mismo sentido, se ha señalado que el mercado laboral español no resulta igualitario para ambos sexos, y que existen claras diferencias en cuestiones de salario, desarrollo y promoción profesional en perjuicio de las mujeres (Agulló \& Ovejero, 2000; Artazcoz, Escribá-Agüir \& Cortés, 2004; Peinado, 1998).

Otra posible explicación a las diferencias de género nos la ofrece Salin (2003a), quien afirma que aquellos individuos con menor poder social, como por ejemplo las minorías étnicas o las mujeres se pueden sentir mas intimidados y ser más sensibles a los conflictos interpersonales, concibiendo de este modo una mayor proporción de situaciones conflictivas como acoso psicológico. En esta misma línea, las mujeres tenderían a clasificar las conductas en mayor medida como acoso debido a los estereotipos de género y las expectativas. De este modo, Salin encontró que las mujeres describen el acoso como una deliberada estrategia competitiva sin focalizar demasiado en factores individuales, mientras que los hombres tienden a ver a las víctimas como débiles, poco competentes e inseguras. De hecho, otros estudios muestran que los hombres conciben la incapacidad para manejar las situaciones negativas como un signo de debilidad (Wright \& Smye, 1997).

En cualquier caso, parece que las diferencias individuales son relevantes a la hora de entender el proceso del acoso psicológico, y que como comentan Zapf y Einarsen (2003) ningún modelo conceptual del acoso laboral será satisfactorio sino incluye tanto los factores individuales como los organizacionales. Aunque el acoso es concebido como un problema causado primordialmente por los factores organizacionales, los factores individuales pueden jugar un rol en su desarrollo, como en todos los problemas relacionados con el estrés. De todos modos, la adopción de modelos individuales deber al menos ser cauta pues se puede caer fácilmente en "culpar a la victima" de la situación que le acontece.

No obstante, los resultados presentados y las sugerencias explicativas expuestas deben ser considerados teniendo en cuenta las limitaciones metodológicas, principalmente muestrales, que tiene el trabajo. Una de las limitaciones del presente 
estudio es el bajo número de sujetos disponibles y el bajo porcentaje de respuesta obtenido. A ello se une la forma de recogida de la muestra (mediante delegados de prevención), lo que podría sesgar los resultados. No obstante, este tipo de limitaciones son habituales en los estudios de este tipo (Björkqvist, Österman \& Lagerspetz, 1994b; Einarsen, 2000). Además habría que atender a la metodología cuantitativa transversal utilizada que, aunque tradicionalmente ha proporcionado datos sobre la correlación entre el acoso y diferentes factores del ambiente de trabajo y la prevalencia de diferentes patologías psicosociales, constituye una aproximación estática al estudio del acoso psicológico, y no permite inferencias causales (Einarsen, Raknes \& Matthiesen, 1994), sino la prevalencia del fenómeno en un determinado momento. De este modo, para incrementar el entendimiento los procesos y factores que conducen a la ocurrencia del mobbing deberían promoverse estudios cualitativos, longitudinales (Salin, 2003b) y transculturales (Einarsen, 2000).

\section{REFERÊNCIAS}

Agulló, E. \& Ovejero, A. (2000). Trabajo, individuo y sociedad Perspectivas psicosociales sobre el futuro del trabajo. Madrid: Pirámide.

Aquino, K. \& Bradfield, M. (2000). Perceived victimization in the workplace: the role of situational factors and victim characteristics. Organization Science, 11(5), 525-537.

Artazcoz, L., Escribá-Agüir, V. \& Cortés, I. (2004). Género, trabajos y salud en España. Gaceta Sanitaria, 18(2), 24-35.

Baron, R. \& Neumann, J. (1998). Workplace aggression-the iceberg beneath the tip of workplace violence: evidence on its forms, frequency and targets. Public Administration Quartely, 21, 446-464.

Björkqvist, K., Österman, K. \& Hjelt-Bäck, M. (1994a). Aggression among university employees. Aggressive Behavior, 20, 173-184.

Björkqvist, K., Österman, K. \& Lagerspetz, K.M.J. (1994b). Sex differences in covert aggression among adults. Aggressive Behaviour, 20, 27-33.

Brodsky, C.M. (1976). The harassed worker. Toronto: Lexington Books/DC Heath and Company.

Comrey, A. L. (1973). A first course in factor analysis. Nueva York: Academic Press.

Einarsen, S. (2000). Harassment and bullying at work: a review of the Scandinavian approach. Aggression and Violent Behavior, 4(5), 379-401.

Einarsen, S. \& Skogstad, A. (1996). Bullying at work: epidemiological findings in public and private organizations. European Journal of Work and Organizational Psychology, 5, 185-201.
Einarsen, S., Raknes, B.I. \& Matthiesen, S.B. (1994). Bullying and harassment at work and their relationships to work environment quality: An exploratory study. European Work and Organizational Psychology, 4(4), 381-401.

Groeblinghoff, D. \& Becker, M. (1996). A case of study of mobbing and the clinical treatment of mobbing victims. European Journal of Work and Organizational Psychology, 5(2), 277-294.

Halford, S. \& Leonard, P. (2001). Gender, Power and Organisations. Basingtoke: Palgrave.

Hoel, H. \& Cooper, C. (2000). Destructive conflict and Bullying at work.

ManchesterSchool of Management. University of Manchester Institute of Science and Technology.

Hoel, H. \& Salin, D. (2003). Organizational antecedents of bullying. En S. Einarsen, H.

Hoel, D. Zapf \& C. Cooper (Org.), Bullying and Emotional Abuse in the Workplace: International Perspectives in Research and Practice (pp. 203-218). London: Taylor \& Francis.

Hofstede, G. (1980). Cultures Consequences: International differences in work related values. Newbury Park, CA: Sage.

Leymann, H. (1986). Vuxenmobbning: om psykiskt våld $i$ arbetslivet. Lund: Studentlitteratur.

Leymann, H. (1992). Mobbing at Swedish work places-a study of 2428 individuals: Frequencies. Stockholm: Arbetarskyddsstyrelsen.

Leymann, H. (1996). Mobbing. La persecution au travail. Paris: Du Seuil.

Leymann, H., \& Gustafsson, B. (1984). Psychological violence at work places. Two explorative studies. Stockholm: Arbetarskyddsstyrelsen.

Matthiesen, S. B. \& Einarsen, S. (2001). MMPI-2 configurations among victims of bullying at work. European Journal of work and organizational Psychology, 10(4), 467484.

McCarthy, P.; Sheehan, M. \& Wilkie, D. (1995). Bullying: from backyard to boardroom. Alexandria: Millennium Books.

Mikkelsen. E. G. \& Einarsen, S. (2001). Bullying in Danish work-life: Prevalence and health correlates. European Journal of Work and Organisational Psychology, 10(4), 393-413.

Moreno-Jiménez, B, Garrosa, E., Galindo, C., San Julián, L., Rodríguez, R., Morante, M. E., \& Losada, M. M. (2002). Mobbing Manual. Transportes y comunicaciones de la Comunidad de Madrid. Madrid: UGT.

Moreno-Jiménez, B., Rodríguez-Muñoz, A., Garrosa Hernández, E., Morante, M. E. \& Rodríguez, R. (2004). Evaluación del acoso psicológico en el trabajo: desarrollo y estudio exploratorio de una escala de medida. (En prensa).

Moreno-Jiménez, B., Rodríguez-Muñoz, A., Morante, M. E. \& Rodríguez, R. (2004). The relevance of organizational factors on the occurrence of mobbing. Psychology \& Health, 19 (supplement), 118.

Niedl, K. (1995). Mobbing/ bullying at the work place. München: Rainer Hampp Verlag.

Nunnaly, J. C. \& Berstein, I. H. (1994, 3 ed.). Psychometric theory. New York: McGraw-Hill. 
Peinado, A. (1988). La discriminación salarial de la mujer en el mercado de trabajo. Madrid: Ministerio de Trabajo y Seguridad Social.

Piñuel, I. (2001). Mobbing. Como sobrevivir al acoso psicológico en el trabajo. Madrid: Sal Térrea.

Rayner, C. (1997). The incidence of workplace bullying. Journal of Community and Applied Social Psychology, 7, 199208.

Rayner, C., Hoel, H. \& Cooper, C. L. (2002). Workplace bullying. What we know, who is blame, and what can we do? New York: Taylor \& Francis.

Salin, D (2001). Prevalence and forms of bullying among business professionals: a comparison of two different strategies for measuring bullying. European Journal of Work and Organizational Psychology, 10(4), 425-441.

Salin, D. (2003a). The significance of gender in the prevalence, forms and perceptions of workplace bullying. Nordiske Organisasjonstudier, 5(3), 30-50.

Salin, D (2003b). Ways of explaining workplace bullying: A review of enabling, motivating and precipitating structures and processes in the work environment. Human Relations, 56(10), 1213-1232.

Scott, J. (1988). Gender and the politics of history. New York: Columbia University Press.
Thylefors, I. (1987). Syndabockar - om utstötning och mobbning i arbetslivet. Natur och Kultur. Stockholm.

Vartia, M. (1996). The sources of bullying - psychological work environment and organizational climate. European Journal of Work and Organizational Psychology, 5(2), 203214.

Wright, L. \& Smye, M. (1997). Corporate abuse: How Learn and Mean Robs people and Profit. New York: Simon and Schuster.

Zapf, D. \& Einarsen, S. (2003). Individual antecedents of bullying. En S. Einarsen, H. Hoel, D. Zapf \& C. Cooper (Org.), Bullying and Emotional Abuse in the Workplace: International Perspectives in Research and Practice (pp.165-184). London: Taylor \& Francis.

Zapf, D., Knorz, C. \& Kulla, M. (1996). On the relationship between mobbing factors, and job content, social work environment and health outcomes. European Journal of Work and Organizational Psychology, 5(2), 215-237.

Recebido em 04/10/2004 Aceito em 24/01/2001
Endereço para correspondência:
Bernardo Moreno-Jiménez, Facultad de Psicología (UAM), Ciudad Universitaria de Cantoblanco. Carretera de Colmenar Viejo, Km. 15, 28049, Madrid-España. E-mail: bernardo.moreno@uam.es 